\title{
RISCO DAS PLANTAS MEDICINAIS NA GESTAÇÃO: UMA REVISÃO DOS DADOS DE ACESSO LIVRE EM LÍNGUA PORTUGUESA
}

\author{
Letícia Englerth Gorril ${ }^{1}$ \\ Ezilda Jacomassi ${ }^{2}$ \\ Sidney Edson Mella Junior ${ }^{1}$ \\ Paulo Roberto Dalsenter ${ }^{3}$ \\ Arquimedes Gasparotto Junior ${ }^{2,4^{*}}$ \\ Emerson Luiz Botelho Lourenço ${ }^{2}$
}

GORRIL, L. E.; JACOMASSI, E.; MELLA JUNIOR, S. E.; DALSENTER, P. R.; GASPAROTTO JUNIOR, A.; LOURENÇO, E. L. B. Risco das plantas medicinais na gestação: uma revisão dos dados de acesso livre em língua portuguesa. Arq. Cienc. Saúde UNIPAR, Umuarama, v. 20, n. 1, p, 67-72, jan./abr. 2016.

RESUMO: A gestação é um período único e especial na vida de cada mulher, no qual ocorre uma série de modificações no organismo feminino, tanto do ponto de visto físico como do ponto de vista psíquico, que começam na primeira semana e continuam durante todo o período de gestação. Essas modificações ocasionam uma série de desconfortos (dores musculares, enjoos, vômitos, e constipação) que interferem no estado físico e emocional da gestante. Na busca por aliviá-las muitas gestantes buscam consumir produtos de origem natural por acreditarem que eles não fazem mal. Nessa busca, a crença de que é "natural" é sinônimo de "seguro" tornam o consumo de plantas medicinais atraente para muitas gestantes, que ao consumirem esses produtos, muitas vezes, sem orientação médica ou farmacêutica, acreditam não existir riscos ao embrião/feto e para si mesmas. Isso faz com que o uso medicinal de plantas seja comum na gestação. Porém, existem evidências científicas que muitas substâncias presentes nas plantas medicinais oferecem riscos durante a gestação. Neste contexto, o presente estudo teve por objetivo investigar, por meio de uma revisão de literatura em bases de acesso livre e em língua portuguesa, quais as espécies que podem acarretar algum risco durante a gestação. A literatura disponível para a população em geral evidenciou que diversas espécies são capazes de oferecer risco durante a gestação por apresentarem potencial embriotóxico, teratogênico e abortífero.

PALAVRAS-CHAVE: Abortivo. Gestante. Plantas Medicinais.

\section{RISK OF MEDICINAL PLANTS IN PREGNANCY: A REVIEW OF FREE ACCESS DATA IN PORTUGUESE}

ABSTRACT: Pregnancy is a unique and special time in every woman's life, in which there is a number of physical and psychological changes in the female body, starting from the first week and continuing throughout the entire pregnancy. These changes cause a lot of discomfort (muscle aches, nausea, vomiting, and constipation) interfering in the physical and emotional state of the mother. In the search for relieving such pains, many pregnant women seek to use natural products because of their belief that they do no harm. In this quest, the belief of what is "natural" is a synonym to "safe" make the consumption of such products attractive to many pregnant women, frequently without any medical or pharmaceutical advice, believing there is no risk to the embryo/fetus and for herself. However, there is scientific evidence that many substances in medicinal plants pose risks during pregnancy. In this context, this study aimed to investigate, through a literature review in open access databases and in Portuguese, which species can entail some risk during pregnancy. The literature data available to the general population showed that several species are able to offer risk during the pregnancy because they have embryotoxic, teratogenic and abortive potential.

KEYWORDS: Abortifacient. Medicinal Plants. Pregnant.

\section{Introdução}

A gestação é um período único e especial na vida de cada mulher (FRIGO et al., 2013), no qual ocorre o crescimento e desenvolvimento de um ou mais embriões, dentro do seu organismo, em um período de tempo que varia de 38 à 42 semanas contadas após o último ciclo menstrual.

Durante a gestação, ocorre uma série de modificações no organismo feminino, tanto do ponto de vista físico (ganho de peso, alterações posturais, enjoos, flatulência, constipação e alterações hormonais) como do ponto de vista emocional (ansiedade, depressão, etc), que começam na primeira semana de gestação e que perduram por todo o período gestacional (HAMERSKI, 2003). Essas modificações têm por finalidade a adaptação do organismo, a manutenção e o desenvolvimento normal da gestação (SILVA, 2008).

Durante a gestação é comum que haja a restrição do uso de determinados medicamentos por indicação médica e farmacêutica que poderiam causar riscos durante a gestação. Isto leva muitas gestantes a buscarem em produtos naturais a solução para atenuar alguns sintomas advindos da gestação (náuseas, vômitos, constipação, azia, etc) e alguns problemas de saúde que podem surgir, como gripes e resfriados, por acreditarem que eles não farão mal a sua saúde e a saúde do seu embrião/feto. Na crença de que o que é "natural" é sinônimo de "seguro", faz com que muitas gestantes recorram ao uso de plantas medicinais para tratar diversos sintomas e enfermidades que podem surgir durante o período gestacional (RANGEL; BRAGANÇA, 2009).

Todavia, existem evidências científicas de que muitas substâncias existentes em algumas plantas medicinais, de uso comum pela população, oferecem riscos à gestação por terem potencial embriotóxico, teratogênico ou abortífero (TABACH et al., 2009); cuja as informações científicas e

DOI: https://doi.org/10.25110/arqsaude.v20i1.2016.5515

${ }^{1}$ Centro Universitário Unicesumar, 87050-900, Maringá, PR, Brasil;

${ }^{2}$ Universidade Paranaense, Programa de Mestrado Profissional em Plantas Medicinais e Fitoterápicos na Atenção Básica, 87502-210, Umuarama, PR, Brasil; ${ }^{3}$ Universidade Federal do Paraná - Departamento de Pós Graduação em Farmacologia, CX. Postal 19031, 81531980 Curtiba, PR, Brasil;

${ }^{4 *}$ Universidade Federal da Grande Dourados, Faculdade de Ciências da Saúde, Cx. Postal 533, 79804-970 Dourados, MS, Brasil. arquimedesgasparotto@ gmail.com 
populares sobre o seu consumo seguro pelas gestantes são escassas ou inexistentes (CLARKE et al., 2007).

A falta de conhecimento sobre os efeitos embriotóxico, teratogênico e abortífero que algumas plantas medicinais podem apresentar, em associação com o seu uso indiscriminado durante a gestação, evidência a necessidade da realização de estudos que investiguem quais plantas medicinais oferecem risco durante este período, e que este conhecimento seja repassado para profissionais da área da saúde e para as próprias gestantes. Dessa forma, analisando a relação risco-benefício ao indicarem o uso plantas medicinas para gestantes e para que estas saibam os riscos que estão sendo expostas.

Neste contexto, o presente estudo teve como objetivo investigar os riscos do uso de plantas medicinas durante a gestação mediante a realização de um estudo de revisão de literatura, utilizando bases de dados de acesso livre e em língua portuguesa, evidenciando as espécies vegetais que podem acarretar algum risco durante este período.

\section{Desenvolvimento}

Este trabalho trata-se de um estudo exploratório, descritivo, de natureza qualitativa, apresentado sobre a forma de revisão de literatura e que teve como questão norteadora: Quais as espécies medicinais oferecem risco durante a gestação?

A busca por publicações científicas foi realizada no período compreendido entre outubro e novembro de 2014 sob forma de consultas realizadas por intermédio da internet a publicações científicas disponibilizadas na "Scientific Electronic Library Online" (SCIELO), Google Acadêmico e acervo pessoal dos autores.

A pesquisa por publicações ocorreu a partir dos seguintes critérios de inclusão: artigo completo publicado no período de 2001 a 2014, em português, com disponibilidade de consulta do artigo na íntegra e de forma gratuita pela internet, mimetizando uma busca realizada por gestantes e interessados pelo tema.

Para a localização das publicações utilizou-se os seguintes descritores cruzados: 1) plantas medicinais, 2) ervas medicas; 3) gravidez, 4) gestação; 5) fitoterápicos e 6) riscos, na forma para "todos os índices" e "todas as fontes", disponível no banco de dados da SCIELO e com busca de modo integrado no Google acadêmico.

A pesquisa gerou 35 artigos, sendo 22 disponibilizados na íntegra. Dessa forma, foram selecionadas as 22 publicações e em seguida, os dados foram acessados, salvos e disponibilizados para leitura e seleção das particularidades que englobaram a pesquisa de forma que o leitor possa identificar as características reais dos estudos revisados e quais espécies podem acarretar algum risco durante o período de gestação.

As plantas medicinais têm uso descrito por praticamente todos os povos existentes à milênios (MENGUE et al., 2001; RODRIGUES et al., 2011). A Organização Mundial da Saúde (OMS) estima que aproximadamente $80 \%$ da população mundial, utilizam plantas medicinais como forma de tratamento aos problemas de saúde (VEIGA JUNIOR; PINTO; MACIEL, 2005; MACENA et al., 2012; SOUZA et al., 2013).

Observações populares feitas ao longo dos anos tem mantido em voga o consumo de plantas medicinais como recurso terapêutico e validado as informações científicas a respeito delas. Como recurso terapêutico, as plantas medicinais podem ter o seu consumo classificado como caseiro ou industrial. $\mathrm{O}$ uso caseiro das plantas medicinais frequentemente se dá sob a forma de chás, xaropes, infusões e garrafadas (MACEDO, OSHIIWA; GUARIDO, 2007; BRASIL, 2012).

$\mathrm{Na}$ indústria farmacêutica as plantas medicinais cujos princípios ativos são reconhecidos e tem eficácia terapêutica comprovada são utilizados na composição química de medicamentos alopáticos e homeopáticos, e comercializados sob a forma de compostos fitoterápicos (BRASIL, 2012).

Outro fato a se destacar a respeito do consumo de plantas medicinais é o fato de que muitas dessas plantas podem ser obtidas pelas pessoas em seus próprios domicílios (em jardins e hortas) sem que haja a necessidade de irem a um comércio de produtos naturais ou farmácias para adquiri-las (EMERENCIANO; ARAÚJO, 2010). Isto faz com que boa parte dessas plantas, seja de fácil acesso, baixo custo, que não necessitem de receituário médico para aquisição e consumo, e que muitas vezes sejam consumidas de modo indiscriminado com base em conhecimentos populares sem comprovação científica, ou conhecimento a respeito dos seus efeitos adversos.

Clarke et al. (2007) e Silva et al. (2012) relatam que apesar das plantas medicinais serem utilizadas a milênios, foi somente no início da última década do século passado que o interesse da comunidade científica sobre as plantas medicinais e o aumento do seu consumo pela população, fez com que a Secretaria de Vigilância Sanitária (SVS) do Ministério da Saúde (MS), regulamentasse o uso das plantas medicinais e fitoterápicos no Brasil. Isto, mediante a publicação da Portaria n. ${ }^{\circ} 6$ de 1995 da SVS/MS, reformulada pela Portaria n. ${ }^{\circ}$ 1029 de 1998 e substituída pela RDC n. ${ }^{\circ} 48$ de 16 de março de 2004. Tal regulamentação passou a exigir que para haver a comercialização de plantas medicinais e de fitoterápicos pela indústria e comércio farmacêutico brasileiro, seria necessário que tais produtos ao ser comercializados tivessem sua eficácia e segurança comprovadas, inclusive para o uso durante a gravidez e lactação caso a mesma possua indicação para este fim.

Conforme relatam Faria et al. (2004) durante a gestação é comum a utilização de plantas medicinais para diversos fins, sendo o seu uso mais comum relacionado ao tratamento de problemas respiratórios (como gripes e resfriados), má digestão e como calmante.

Pelo fato de existirem estudos científicos que evidenciam que algumas espécies medicinais apresentam efeito embriotóxico, teratogênico e abortivo, seu uso durante a gestação deve ser restrito ou impedido. Pois assim como ocorre com alguns medicamentos sintéticos, o consumo de plantas medicinais por gestantes deve levar em conta a relação risco-benefício. A Tabela 1 mostra as espécies medicinais que oferecem riscos durante a gestação. Nesta tabela é possível observar o nome científico, o nome popular, família de cada planta e o relato do risco no período de gestação. 
Tabela 1: Relação das espécies medicinais que oferecem riscos durante o período gestacional

\begin{tabular}{|c|c|c|c|}
\hline Nome científico & Nome Popular & Família & Risco à gestação \\
\hline Abrus precatorius L. & Ervilha-do-rosário & Fabaceae & Abortiva \\
\hline Achyrocline satureioides (Lam.) DC. & Marcela & Asteraceae & Abortiva \\
\hline Aesculus hippocastanum L. & Castanha-da-índia & Sapindaceae & Abortiva \\
\hline Aristolochia triangularis (Cham) & Cipó-mil-homens & Aristolochiaceae & Abortiva \\
\hline Artemisia absinthium L. & Losna & Asteraceae & Abortiva \\
\hline Artemisia verlotorum Lamotte & Infalivina & Asteraceae & Abortiva \\
\hline Artemisia vulgaris L. & Artemisia & Asteraceae & Abortiva \\
\hline Astronium urundeuva (Allemão) Engl. & Aroeira & Anacardiaceae & Abortiva \\
\hline Baccharis trimera (Less.) DC. & Carqueja & Asteraceae & Abortiva \\
\hline Brunfelsia uniflora (Pohl) D. Don & Manacá-de-jardim & Solanaceae & Abortiva \\
\hline Casearia sylvestris Sw. & Guaçatonga & Salicaceae & Abortiva \\
\hline Catharanthus roseus (L.) G. Don & Vinca & Apocynaceae & Abortiva \\
\hline Chamomilla recutita (L.) Rauschert & Camomila & Asteraceae & Emenagoga e abortiva \\
\hline Chenopodium ambrosioides L. & Erva-de-santa-maria & Amaranthaceae & Abortiva \\
\hline Cinnamomum verum J. Presl & Canela & Lauraceae & Abortiva \\
\hline Coffea arabica $\mathrm{L}$. & Café & Rubiaceae & Abortiva \\
\hline Coutarea hexandra (Jacq.) K. Schum. & Quina-quina & Rubiacae & Abortiva \\
\hline Cunila fasciculata Benth. & Poejo & Lamiaceae & Abortiva \\
\hline Cunila galioides Benth. & Poejinho & Lamiaceae & Abortiva \\
\hline Cunila menthoides Benth. & Poejo-de-folha-grande & Lamiaceae & Abortiva \\
\hline Cymbopogon citratus (DC.) Stapf & Capim-cidreira & Poaceae & Relaxante uterino \\
\hline Dorstenia brasiliensis Lam. & Carapiá & Moraceae & Abortiva \\
\hline Equisetum giganteum L. & Cavalinha & Equisetaceae & Abortiva \\
\hline Foeniculum vulogare L. & Erva-doce & Apiaceae & $\begin{array}{c}\text { Relaxante uterino e } \\
\text { abortiva }\end{array}$ \\
\hline Gossypium barbadense L. & Algodoeiro & Malvaceae & Abortiva \\
\hline Himatanthus drasticus (Mart.) Plumel & Carapanaúba & Apocynaceae & Abortiva \\
\hline Himatanthus lancifolius (Müll. Arg.) Woodson & Agoniada & Apocynaceae & Abortiva \\
\hline Jatropha curcas L. & Pinhão-manso & Euphorbiaceae & Abortiva \\
\hline Jatropha gossypiifolia $\mathrm{L}$. & Pinhão-roxo & Euphorbiaceae & Abortiva \\
\hline Kalanchoe brasiliensis Cambess. & Saião & Crassulaceae & Abortiva \\
\hline Laurus nobilis L. & Louro & Lauraceae & Abortiva \\
\hline Luffa operculata (L.) Cong. & Buchinha & Cucurbitacea & Abortiva e embriotóxica \\
\hline Mentha piperita $\mathrm{L}$. & Hortelã-pimenta & Lamiaceae & Abortiva \\
\hline Mentha sp & Hortelã & Lamiaceae & Abortiva \\
\hline Momordica charantia L. & Melão-do-campo & Cucurbitaceae & Abortiva \\
\hline Newbouldia laevis (P. Beauv.) Seem. & Capim-de-oxalá & Bignoniaceae & Abortiva \\
\hline Petiveria alliacea $\mathrm{L}$. & Guiné & Phytolaccaceae & Abortiva \\
\hline Pimpinella anisum L. & Erva-doce & Apiaceae & Abortiva \\
\hline Peumus boldus Molina & Boldo-do-chile & Monimiaceae & Abortivo e teratogênica \\
\hline Plectranthus barbatus Andrews & Boldo-da-terra & Lamiaceae & Abortiva \\
\hline Pluchea sagittalis (Lam.) Cabrera & Quitoco & Asteraceae & Abortiva \\
\hline Rosmarinus officinalis L. & Alecrim & Laminaceae & Emenagoga e abortiva \\
\hline Ruta graveolens $\mathrm{L}$. & Arruda & Rutaceae & Abortiva \\
\hline Schinus terebinthifolius Raddi & Aroeira & Anacardiaceae & Abortiva \\
\hline Senna alexandrina Mill. & Sene & Fabaceae & Abortiva \\
\hline Strychnos pseudoquina A. St.-Hil. & Quina-verdadeira & Loganiaceae & Abortiva \\
\hline
\end{tabular}




\begin{tabular}{l|c|c|c}
\hline Symphytum officinale L. & Confrei & Boraginaceae & Abortiva \\
\hline Syzygium aromaticum (L.) Merr. \& L.M. Perry. & Cravo & Myrtaceae & Abortiva \\
\hline Tabebuia heptaphylla (Vell.) Toledo & Ipê-roxo & Bignoniaceae & Abortiva \\
\hline Tropaeolum majus L. & Chaguinha & Tropaeolaceae & Abortiva \\
\hline Vernonia condensata Baker & Figatil & Asteraceae & Abortiva \\
\hline Xanthium cavanillesii Schouw & Carrapixo & Asteraceae & Abortiva \\
\hline Zanthoxylum caribaeum Lam. & Espinho-cheiroso & Rutaceae & Abortiva \\
\hline
\end{tabular}

Fonte: Adaptado de Mengue et al. (2001), Clarke et al. (2007) e Silva et al. (2012).

Considerando os dados obtidos neste estudo, pode-se observar que diversos trabalhos científicos descrevem informações sobre a ação abortiva de algumas espécies vegetais comumente utilizadas na medicina popular. Nos estudos de Vendruscolo e Mentz (2006) e Silva et al. (2012) são relatados que os extratos aquosos de Ruta graveolens (arruda) pode interferir no desenvolvimento embrionário pré-implantação, no transporte do embrião até o útero e induzir um aumento de embriões anormais nas fases de mórula e blastocisto. Além disso, as folhas de Tropaeolum majus (chaguinha) na forma de extrato hidroalcoólico podem promover perdas pré - implantes devido ao aumento dos níveis de estradiol, redução do peso dos fetos e anomalias renais em estudos realizados com animais de experimentação ( LOURENÇO; GASPAROTTO JUNIOR; DALSENTER, 2014). Ataíde et al. (2007) relatam que a infusão das folhas secas de Peumus boldus (boldo-do-chile) durante a gestação provoca contração uterina e risco de aborto. Luffa operculata (buchinha), rica em glicoproteínas, possui importante ação embriotóxica e está entre as plantas mais utilizadas como abortivas no Brasil (MENGUE et al., 2001; SILVA et al., 2012).

Ainda em relação as espécies vegetais que interferem na gestação e no desenvolvimento embrionário e fetal, Silva et al. (2012) destacam que a Aristolochia triangularis (cipó-mil-homens) possui importante ação sobre o útero e não deve ser usada na gestação por ser potencialmente abortiva. As sementes de Momordica charantia (melão-do-campo) são ricas em glicoproteínas com ação inibitória sobre a multiplicação celular do endométrio e miométrio, com consequente efeito abortivo (CLARKE et al., 2007; SILVA et al., 2012).

Compondo o grupo de plantas medicinais com efeito abortivo, Castro et al. (2004) destacam a canela (Cinnamomum verum), o cravo (Syzygium aromaticum), as folhas do café (Coffea arabica), o capim-de-oxalá (Newbouldia laevis), a quina-verdadeira (Strychnos pseudoquina), o quitoco (Pluchea sagittalis) e a aroeira (Schinus terebinthifolius), consumidos sob a forma de chá. Faria et al. (2004) relatam ainda que a camomila (Chamomilla recutita), a erva-doce (Pimpinella anisum) e o capim-limão (Cymbopogon citratus), possuem ação terapêutica reconhecida como calmante e antiespasmódica. Entretanto, o consumo é contraindicado durante a gestação por provocar o relaxamento do útero, estimular o sangramento e o aborto espontâneo.

Ruiz et al. (2008) e Araujo e Macedo (2011) relatam que o espinho-cheiroso (Zanthoxylum caribaeum), o figatil (Vernonia condensata), a castanha-da-índia (Aesculus hippocastanum) e a carqueja (Baccharis trimera) também são contraindicados na gestação pelo seu potencial abortivo. Além disso, o uso sistêmico de algumas espécies medicinais pode ser contraindicado durante o período gestacional devido seu potencial teratogênico. Isto porque, os princípios ativos de certas plantas são capazes de passarem pela barreira placentária, podendo afetar a formação do feto (FARIA et al., 2004).

Assim, as informações técnicas disponíveis ainda são insuficientes para a maioria das espécies medicinais de modo a garantir a sua qualidade, a sua eficácia e a sua segurança de uso das mesmas durante o período gestacional (FIRMO et al., 2011). O fato é que por se tratar de plantas as espécies medicinais podem ser facilmente obtidas pelas pessoas em seus próprios quintais, como em jardins e hortas, sem que haja a necessidade de ir a um comércio de produtos naturais ou farmacêuticos para adquiri-las (EMERENCIANO; ARAÚJO, 2010). Isto faz com que boa parte das plantas apresentadas na Tabela 1 sejam frequentemente utilizadas pela população como forma de tratamento para diversas enfermidades e sintomas clínicos, devido ao fácil acesso, baixo custo e uso baseado em conhecimentos populares, sem comprovação científica, ou sem conhecimento a respeito dos efeitos adversos e riscos durante a gestação.

Entre as gestantes é reportado na literatura que as plantas medicinais apresentadas na Tabela 1 são consumidas frequentemente com base nas indicações terapêuticas fornecidas por familiares. Um estudo realizado por Faria et al. (2004) evidenciou que aproximadamente $70 \%$ das gestantes referem conhecer as indicações terapêuticas das plantas medicinais que usam e que cerca de $30 \%$ não conhecem os riscos que o consumo dessas plantas podem acarretar.

A literatura científica disponível sobre o uso das plantas medicinais durante a gestação relata que muitas espécies são de uso corriqueiro pela população, destacando-se a Ruta graveolens (arruda), Salvia officinalis (salvia), Punica granatum (romã), Datura suaveolens (beladona), Allium sativum (alho), Aloe ferox (aloe), Angelica archangelica (angélica), Arnica montana (arnica), Cinnamomum canphora (cânfora), Symphitum officinale (confrei), Eucaliptus globulus (eucalipto), Rosmarinus officinalis (alecrim), Zingiber officinalis (gengibre), Cassia angustifolia, Hibiscus rosa-sinensis (hibiscus), Baccharis trimera (carqueja), Реuтus boldus (boldo-do-chile), Luffa operculata (buchinha), Artemisia vulgaris (artemísia) e Hibiscus sabdariffa (hisbiscus) (SOUZA et al., 2013).

Apesar dessas espécies serem contraindicadas no período gestacional devido aos riscos embriotóxico, teratogênico e abortivo elas são comumente consumidas pelas gestantes devido ao fato de serem economicamente mais acessíveis, pois desconhecem seus efeitos indesejados durante o período gestacional e por acreditarem que o consumo de produtos naturais é menos nocivo ao organismo materno e fetal (CLARKE et al., 2007; RODRIGUES et al., 2011; SILVA et 


\section{al., 2012; FARIA et al., 2004).}

Segundo Rodrigues et al. (2011) os efeitos mais preocupantes do uso indiscriminado de plantas medicinais por gestantes é de fato o efeito teratogênico e embriotóxico, pois os metabólitos secundários presentes nestas plantas podem atravessar a barreira placentária, chegar ao embrião ou feto, e colocar em risco o seu desenvolvimento normal.

Apesar de todas as informações disponíveis, existem controvérsias sobre o efeito teratogênico, embriotóxico e abortivo das plantas medicinais, não apenas pela falta de comprovação científica, mas também pelas interações com outros medicamentos, procedência dessas plantas, tempo de gestação, forma de preparo e uso (FARIA et al., 2004; RODRIGUES et al., 2011). Dessa forma, estudos pré-clínicos controlados devem ser conduzidos para avaliar o real impacto do consumo das plantas medicinais durante todo o processo de embriogênese e no período gestacional.

\section{Conclusão}

Mediante a realização do presente estudo foi possível constatar que diversas plantas medicinais oferecem riscos durante o período gestacional. Os principais riscos aos quais as gestantes estão expostas durante o consumo indiscriminado de plantas medicinais estão relacionados ao potencial embriotóxico, teratogênico e abortivo. Existem fortes evidências científicas de que Ruta graveolens (arruda), Peumus boldus (boldo-do-chile) e Luffa operculata (buchinha) são as espécies que representam maior risco durante a gestação, pois estão entre as mais citadas nas publicações científicas dentro deste contexto. Por fim, conclui-se a grande necessidade da realização de novos estudos pré-clínicos que investiguem de forma mais aprofundada os riscos causados pelo consumo dessas espécies durante a gestação, especialmente no que diz respeito ao princípio ativo responsável pelo risco, e a dosagem recomendada para o consumo destas plantas durante o período gestacional.

\section{Referências}

ARAUJO, P. S.; MACEDO, A. P. Percepção de risco e conceitos sobre plantas medicinais, fitoterápicos e alopáticos entre gestantes. Revista Baiana de Saúde Pública, v. 35, n. 2, p. 320-333, 2011.

ATAÍDE, R. A. et al. Uso de remédios caseiros por mulheres do Programa Saúde da Família. Revista Enfermagem UFPE, v. 1, n. 2, p. 97-103, 2007.

LOURENÇO, E. L. B.; GASPAROTTO JUNIOR, A.; DALSENTER, P. R. Toxicologia reprodutiva de Tropaeolum majus (chaguinha): riscos do uso das folhas de Tropaeolum majus L. durante a gestação. Curitiba: Novas Edições Acadêmicas, 2014.

BRASIL. Ministério da Saúde. Secretaria de Atenção a Saúde. Departamento de Atenção Básica. Práticas integrativas e complementares: plantas medicinais e fitoterapia na atenção básica. Brasília: Ministério da Saúde, 2012. 156 p.
CASTRO, C. G. S. et al. Uso indicado e uso referido de medicamentos durante a gravidez. Cadernos de Saúde Pública, v. 20, n. 1, p.73-S82, 2004.

CLARKE, J. H. R. et al. Um alerta sobre o uso de produtos de origem vegetal na gravidez. Infarma, v. 19, n. 1-2, p. 41-48, 2007.

EMERENCIANO, C. S.; ARAUJO, A. P. S. Fitoterapia na promoção de saúde e o seu uso na prática clínica da acupuntura. UNINGÁ Review, v. 1, n. 1, p. 26-33, 2010.

FARIA, P. G. et al. O diálogo com gestantes sobre plantas medicinais: contribuições para os cuidados básicos de saúde. Acta Scientiarum, Health Sciences, v. 26, n. 2, p. 287-294, 2004.

FIRMO, W. C. A. et al. Contexto histórico, uso popular e concepção cientifica sobre plantas medicinais. Cadernos de Pesquisa, v.18, n. especial, 2011.

FRIGO, J. et al. Assistência de enfermagem e a perspectiva da mulher no trabalho de parto e parto. Cogitare

Enfermagem, v. 18, n. 4, p.761-766, 2013.

HAMERSKI, L. M. Mulheres em seu primeiro parto: relatando as vivências, expectativas e sentimentos. 2003. 65 f. Trabalho de Conclusão de Curso (Graduação em Enfermagem) - Universidade Regional do Noroeste do Estado do Rio Grande do Sul, Ijuí, 2003.

MACEDO, A. F.; OSHIIWA, M.; GUARIDO, C. F. Ocorrência do uso de plantas medicinais por moradores de um bairro do município de Marília-SP. Revista de Ciências Farmacêuticas Básica e Aplicada, v. 28, n. 1, p. 123-128, 2007.

MACENA, L. M. et al. Plantas medicinais utilizadas por gestantes atendidas na unidade de saúde da família (USF) do bairro COHAB Tarumã no município de Tangará da Serra, Mato Grosso. BioFar, v. 7, n. 1, p. 143-155, 2012.

MENGUE, S. S. et al. Uso de plantas medicinais na gravidez. In: SANSEVERINO, M. T. V.; SORITZER, D. T.; SCHULER-FACCINI, L. Manual de teratogênese. Porto Alegre: Universidade UFRGS, 2001. p. 423-450.

RANGEL, M.; BRAGANCA, F. C. R. Representações de gestantes sobre o uso de plantas medicinais. Revista Brasileira de Plantas Medicinais, v. 11, n. 1, p. 100-109, 2009.

RODRIGUES, H. G. et al. Efeito embriotóxico, teratogênico e abortivo de plantas medicinais. Revista Brasileira de Plantas Medicinais, v. 13, n. 3, p. 359-366, 2011.

RUIZ, A. L. T. G. et al. Farmacologia e toxicologia de Peumus boldus e Baccharis genistelloides. Revista Brasileira de Farmacognosia, v. 18, n. 2, p. 295-300, 2008. 
SILVA, R. C. et al. Plantas medicinais utilizadas na saúde da mulher: riscos na gravidez. Diálogos \& Ciência, v. 1, n. 32, p. 20-28, 2012.

SILVA, S. C. F. Ansiedade da mulher durante o último trimestre de gravidez. 2008. 65 f. Trabalho de Conclusão de Curso (Graduação em Enfermagem) - Universidade Fernando Pessoa, Porto, 2008.

SOUZA, M. N. C.V. et al. Plantas medicinais abortivas utilizadas por mulheres de UBS: etnofarmacologia e análises cromatográficas por CCD e CLAE. Revista Brasileira de Plantas Medicinais, v. 15, n. 4, p. 763-773, 2013.

TABACH, R. Sistema de farmacovigilância em plantas medicinais. Boletim Planfavi, v. 1, n. 10, p. 1-4, 2009.

VEIGA JUNIOR, V. F.; PINTO, A. C.; MACIEL, M. A. M. Plantas medicinais: cura segura? Química Nova, v. 28, n. 3, p. 519-528, 2005.

VENDRUSCOLO, G. S.; MENTZ, L. A. Levantamento etnobotânico das plantas utilizadas como medicinais por moradores do bairro Ponta Grossa, Porto Alegre, Rio Grande do Sul, Brasil. Iheringia Série Botânica, v. 61, n. 1-2, p. 83-103, 2006. 